\title{
Dynamic Behaviors Analysis of a Novel Fractional-Order Chua's Memristive Circuit
}

\author{
Chaojun Wu $\mathbb{D},{ }^{1}$ Qi Zhang $\mathbb{D}^{1},{ }^{1}$ Zhang Liu $\mathbb{D}^{1},{ }^{1}$ and Ningning Yang $\mathbb{D}^{2}$ \\ ${ }^{1}$ School of Electronics and Information, Xi'an Polytechnic University, Xi'an 710048, China \\ ${ }^{2}$ School of Electrical Engineering, Xi'an University of Technology, Xi'an 710048, China
}

Correspondence should be addressed to Ningning Yang; ningning.yang@stu.xjtu.edu.cn

Received 22 May 2021; Accepted 3 July 2021; Published 13 July 2021

Academic Editor: Zenghui Wang

Copyright (c) 2021 Chaojun Wu et al. This is an open access article distributed under the Creative Commons Attribution License, which permits unrestricted use, distribution, and reproduction in any medium, provided the original work is properly cited.

This paper proposed a novel fractional-order Chua's memristive circuit. Firstly, a fractional-order mathematical model of a diode bridge generalized memristor with RLC filter cascade is established, and simulations verify that the fractional-order generalized memristor satisfies the basic characteristics of a memristor. Secondly, the capacitor and inductor in Chua's chaotic circuit are extended to the fractional order, and the fractional-order generalized memristor is used instead of Chua's diode to establish the fractional-order mathematical model of chaotic circuit based on RLC generalized memristor. By studying the stability analysis of the equilibrium point and the influence of the circuit parameters on the system dynamics, the dynamic characteristics of the proposed chaotic circuit are theoretically analyzed and numerically simulated. The results show that the proposed fractional-order memristive chaotic circuit has gone through three states: period, bifurcation, and chaos, and a narrow period window appears in the chaotic region. Finally, the equivalent circuit method is adopted in PSpice to realize the construction of the fractional-order capacitance and inductance, and the simulation of the fractional-order memristive chaotic circuit is completed. The results further verify the correctness of the theoretical analysis.

\section{Introduction}

Memristor is a nonlinear circuit element that describes the relationship between charge and magnetic flux. Its theoretical concept was first proposed by Professor Chua in 1971 [1]. It has electrical characteristics that cannot be achieved by any combination of three basic elements: resistance, capacitance, and inductance. In 2008, HP Labs proved the physical existence of memristor for the first time, responding to Professor Cai's speculation [2]. Since then, the related theoretical research of memristor has entered the practical stage, which has attracted strong attention from researchers [3-6].

The memristor is a nonlinear device, and it is easy to produce abundant chaotic phenomena when combined with other oscillating circuits. As early as 2008, Itoh and Chua replaced Chua's diode with a memristor based on the known Chua's chaotic circuit and obtained a memristive chaotic system [7]. In 2010, Bao Bocheng and others replaced the diode in the Chua's oscillator with a memristor and a negative conductance. By setting different parameters and initial conditions, they showed different dynamic behaviors [8]. Later, Pham proposed a memristive hyperchaotic system and found that the system has hidden attractors [9]. This hyperchaotic system based on memristor has no equilibrium point, but it can still exhibit chaotic phenomena. Wang et al. [10] proposed a new type of memristor-based Wien-bridge oscillator and studied its parameter-independent dynamical behaviors. A hyperchaotic memristive circuit based on the Wien-bridge oscillator structure is proposed in [11]. Chang et al. [12] proposes and analyzes a memristor with coexistent shrinkage hysteresis loop and twin local activity domains and applies it to the classic Chua's circuit to replace the diode. The complex dynamics of the system are analyzed using compound coexistence bifurcation diagram, Lyapunov exponent spectrum, and phase diagram. In recent years, various novel memristive chaotic circuits have been proposed and analyzed, such as a three-order Wien-bridge chaotic circuit without inductor [13], 
a memristive chaotic oscillator with controllable amplitude and frequency [14], and a physical SBT memristor-based chaotic circuit [15]. In addition, researchers have also conducted indepth studies on generalized memristors, such as diode bridgecascaded RLC memristors and a generalized memristive simulator composed of a first-order RC $[16,17]$.

As we all know, almost all electronic devices are not ideal devices. Therefore, there will be deviations when using an ideal model to analyze actual electronic components. With the development of the theory of fractional calculus, Carlson and others proposed the concept of fractional-order capacitance [18]. Nowadays, a large number of studies have shown that fractional-order modeling of the system makes the description of its electrical characteristics more accurate. According to the theory of fractional calculus, a more accurate model can be obtained by extending the memristive chaotic circuit to the fractional order. This makes the frequency and electrical characteristics of the fractional-order memristive chaotic system more valuable for research [19]. Ding et al. [20] proposed a fractional-order memristive Chua's circuit with time delay, which is composed of a passive flux-controlled memristor and a negative conductance in parallel and then analyzed the coexistent multiple stability of the system. Yan et al. [21] conducted a numerical study on the dynamics, coexisting attractors, complexity, and synchronization of the fractional-order memristor-based hyperchaotic system. Petras [22] studied the application of fractional calculus in nonlinear circuits and analyzed the fractional-order equation and numerical solution of Chua's oscillator based on memristor. $\mathrm{Wu}$ et al. [23] proposed an active fractional-order memristor model and analyzed the electrical characteristics of the memristor via power-off plot and dynamic road map. Finally, it is found that the fractional-order memristor has continually stable states, so it is nonvolatile. It is worth mentioning that both fractional calculus theory and memristor are current research hotspots. Therefore, a large number of scholars have concentrated on the research of the chaotic oscillator circuit based on the fractional-order memristor [24-28].

The rest of the paper is organized as follows. Section 2 briefly introduces the fundamental of fractional calculus and constructs a fractional-order generalized memristor composed of a diode bridge and a second-order RLC filter and then proposes a fractional-order memristive chaotic circuit. In Section 3, by studying the stability of the equilibrium points and the influence of system parameters on the bifurcation, the dynamic behavior of the proposed fractional memristive chaotic circuit is theoretically analyzed and numerically simulated. Section 4 realizes the construction of the equivalent circuit of the fractional-order inductor and capacitor and then completes the circuit simulation of the fractional-order memristive chaotic circuit in PSpice. The last section is the conclusion.

\section{Fractional Models of Generalized Memristor and Chua's Memristive Circuit}

2.1. Fractional Calculus Theory. The theory of fractional calculus has been studied for more than 300 years. The operator $D_{t}^{\alpha}$ of fractional calculus is defined as follows:

$$
{ }_{a} D_{t}^{\alpha}= \begin{cases}\frac{\mathrm{d}^{\alpha}}{\mathrm{d} t^{\alpha},} & \alpha>0, \\ 1, & \alpha=0, \\ \int_{a}^{t}(\mathrm{~d} \tau)^{-\alpha}, & \alpha<0,\end{cases}
$$

where $\alpha$ is limited to real numbers, $t$ is the independent variable, and $a$ is the lower boundary of the variable [29].

The definition proposed by Caputo is more convenient for initial conditions and closer to practical applications, so this paper uses Caputo definition. The fractional Caputo derivative of the function $f(t)$ is defined as follows:

$$
{ }_{a}^{C} D_{t}^{\alpha} f(t)=\frac{1}{\Gamma(n-a)} \int_{a}^{t} \frac{f^{(n)}(\tau)}{(t-\tau)^{\alpha-n+1}} \mathrm{~d} \tau, \quad(0 \leq n-1<\alpha \leq n) .
$$

In the formula, $\Gamma(\cdot)$ is the Gamma function and $n \in N$ is an integer.

Under the natural condition of the function $f(t)$, for $\alpha \longrightarrow n$, the Caputo definition becomes the normal $n$-order derivative of the function $f(t)$. The Laplace transform of Caputo fractional derivative is directly calculated by the following formula:

$$
L\left[{ }_{a}^{C} D_{t}^{\alpha} f(t)\right]=s^{\alpha} F(s)-\sum_{k=0}^{n-1} s^{\alpha-k-1} f^{(k)}(0), \quad(n-1<\alpha \leq n) .
$$

2.2. The Fractional-Order Generalized Memristor. Corinto and Ascoli [30] proposed a full-wave rectifier with a secondorder RLC filter with memristive characteristics. On this basis, this paper proposes a new second-order generalized memristor composed of a diode bridge and a parallel RLC filter, and its circuit model is shown in Figure 1.

The mathematical model of the second-order RLC generalized memristor is as follows:

$$
\begin{aligned}
& i_{\text {in }}=f\left(v_{c}, v_{\text {in }}\right)=2 I_{s} e^{-\rho v_{c}} \sinh \left(\rho v_{\text {in }}\right), \\
& \frac{\mathrm{d} x}{\mathrm{~d} t}=F\left(x, v_{\text {in }}\right)=\left[\begin{array}{c}
\frac{2 I_{s}\left(e^{-\rho v_{c}} \cosh \left(\rho v_{\text {in }}\right)-1\right)-i_{L}}{C} \\
\frac{v_{c}-R i_{L}}{L}
\end{array}\right],
\end{aligned}
$$

where $\rho=1 /\left(2 n V_{T}\right)$, and $I_{s}, n$, and $V_{T}$ are the diode reverse saturation current, emissivity, and thermal voltage, respectively. In addition, $v_{\text {in }}$ is the excitation voltage of the generalized memristor, $i_{\text {in }}$ is the input current, and $x=\left[v_{c}, i_{L}\right]^{T}$. The internal function expression of the generalized memristor is as follows:

$$
G_{M}=\frac{i_{\text {in }}}{v_{\text {in }}}=\frac{2 I_{s} e^{-\rho v_{c}} \sinh \left(\rho v_{\text {in }}\right)}{v_{\text {in }}} .
$$




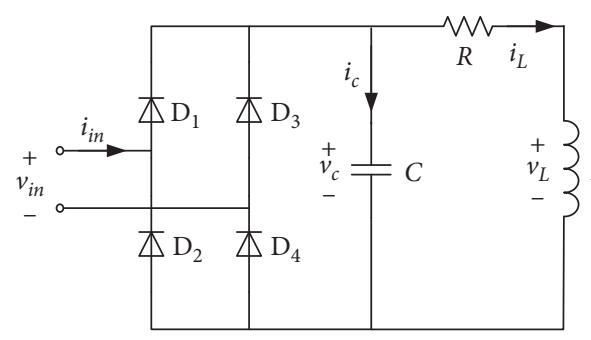

(a)

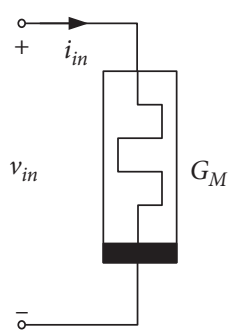

(b)

FIGURE 1: The circuit model of the second-order generalized memristor. (a) Memristive diode bridge and parallel RLC filter and (b) generalized memristor.

According to the theory of fractional calculus, this paper expands the integer-order capacitor and inductor in the generalized memristor to the fractional order to describe its electrical characteristics. The fractional-order circuit model is shown in Figure 2, where $q$ is the order.

Therefore, the unified mathematical model of the fractional-order generalized memristor is as follows:

$$
\left\{\begin{array}{l}
\frac{\mathrm{d}^{q} v_{c}}{\mathrm{~d} t^{q}}=F\left(x, v_{\text {in }}\right)=\left[\begin{array}{c}
\frac{2 I_{s}\left(e^{-\rho v_{c}} \cosh \left(\rho v_{\text {in }}\right)-1\right)-i_{L}}{C^{q}} \\
\frac{v_{c}-R i_{L}}{L^{q}}
\end{array}\right], \\
i_{\text {in }}=f\left(v_{c}, v_{\text {in }}\right)=2 I_{s} e^{-\rho v_{c}} \sinh \left(\rho v_{\text {in }}\right), \\
G_{M}=\frac{i_{\text {in }}}{v_{\text {in }}}=\frac{2 I_{s} e^{-\rho v_{c}} \sinh \left(\rho v_{\text {in }}\right)}{v_{\text {in }}}
\end{array}\right.
$$

The component parameters of the novel fractional-order RLC generalized memristor are as follows: $C=4 \mathrm{nF}$, $L=230 \mathrm{mH}$, and $R=800 \Omega$; the diode parameters are as follows: $I_{s}=2.682 \mathrm{nA}, n=1.836$, and $V_{T}=25 \mathrm{mV}$. The Oustaloup method was used to obtain the approximate transfer function of the fractional-order integral operator [31]. In the frequency domain, the transfer function of a fractional integral operator with order $q$ can be expressed as $F(s)=1 / s^{q}$. According to the Oustaloup approximation technique, transfer functions of different orders at the operating frequency can be obtained. Table 1 gives the approximate transfer functions of the fractional-order integrator $F(s)$ with orders of $0.87,0.9,0.95$, and 0.97 , respectively.

The fractional-order capacitor $C^{q}$ is realized by the equivalent unit circuit in Figure 3. The fractional-order inductance $L^{q}$ is realized by the equivalent circuit in Figure 4.

In the complex frequency domain, the equivalent circuit expression for realizing the fractional-order capacitor $C^{q}$ is equation (7). Tables 2 and 3, respectively, give the calculated values of resistance and capacitance in the equivalent cell circuit under different orders. The calculated values of resistance and inductance in the equivalent circuit of fractional inductor $L^{q}$ under different orders are calculated by formula (8), which are given in Tables 4 and 5, respectively.

$$
\begin{aligned}
& F(s)=\frac{1}{C s^{q}}=R_{\mathrm{in}}+\sum_{k=1}^{n} \frac{\left(1 / C_{k}\right)}{\left(s+\left(1 / R_{k} C_{k}\right)\right)}, \\
& F(s)=\frac{1}{L} s^{-q}=\sum_{k=1}^{n} \frac{1}{L_{k} s+R_{k}}+\frac{1}{R_{\text {in }}} .
\end{aligned}
$$

On the basis of the aforementioned theory, in order to verify whether the proposed novel fractional-order RLC generalized memristor possesses the three essential characteristics of memristor [32], numerical simulation is performed using MATLAB/Simulink software. The input excitation source is $u_{\text {in }}=V_{m} \sin (2 \pi f t) V$, where $f$ is the frequency of the input voltage and $V_{m}$ is the amplitude of the input voltage. When $V_{m}=4 \mathrm{~V}$ and the order $q$ are, respectively, $0.87,0.9,0.95$, and 0.97 , the $i_{\text {in }}-u_{\text {in }}$ curves under different frequencies are shown in Figure 5. It can be seen from Figure 5 that the trajectory in the $i_{\text {in }}-u_{\text {in }}$ plane shrinks at the origin, and the area of the tight hysteresis loop decreases monotonously as the excitation frequency increases. In addition, when the frequency of the input voltage tends to infinity, the tight hysteresis loop shrinks to a nonlinear single-valued function, which proves that the proposed fractional-order generalized memristor satisfies the basic characteristics of the memristor.

In addition, as the input voltage amplitude increases, the area of the tight hysteresis loop will increase, but the shape remains unchanged, as shown in Figure 6(a). In order to analyze the influence of the order on the characteristics of the memristor model, fix $V_{m}=4 \mathrm{~V}, f=1 \mathrm{kHz}$, and $q$ to be $0.87,0.9,0.95$, and 0.97 , respectively, and obtain the voltampere characteristic curves under different orders, as shown in Figure 6(b). It is found that the area of the tight hysteresis loop of the fractional-order generalized memristor decreases monotonously with the increase of the order under the same amplitude and frequency.

2.3. The Fractional-Order Memristive Chaotic Circuit. Yang et al. [33] proposed a chaotic circuit composed of capacitance, inductance, resistance, negative conductance, and nonlinear passive memristor. After realizing the fractional-order generalized memristor, a fractional-order memristive chaotic circuit is established using the proposed 


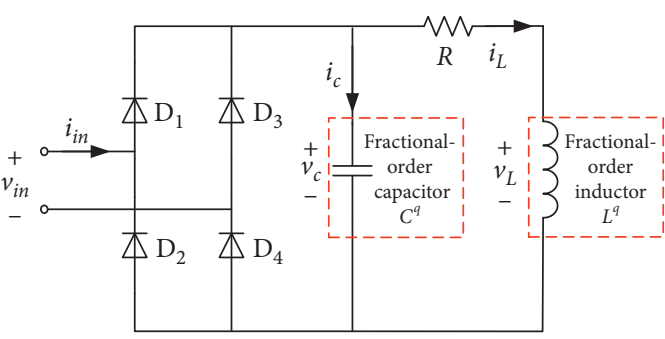

(a)

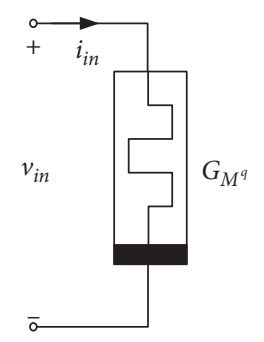

(b)

FIGURE 2: The circuit model of the fractional-order generalized memristor.

novel fractional-order RLC generalized memristor $M^{q}$ instead of Chua's diode, as shown in Figure 7.

$v_{c 1}$ is the voltage across the fractional-order capacitor $C_{1}^{q}$, and $v_{c 2}$ is the voltage across the fractional-order capacitor $C_{2}^{q}$. The current flowing through the fractional-order inductor $L_{1}^{q}$ is defined as $i_{1}$. The voltage across the memristor is $v_{M}$ and the current is $i_{M} \cdot R, L^{q}$, and $C^{q}$ are the internal resistance, fractional-order inductor, and fractional-order capacitor of the generalized memristor, respectively. Therefore, the mathematical model of the fractional-order memristive chaotic system can be written as follows:

$$
\left\{\begin{array}{l}
\frac{\mathrm{d}^{q} v_{c 1}}{\mathrm{~d} t^{q}}=\frac{v_{c 2}-v_{c 1}}{R_{o} C_{1}^{q}}-\frac{i_{1}}{C_{1}^{q}}, \\
\frac{\mathrm{d}^{q} v_{c 2}}{\mathrm{~d} t^{q}}=\frac{v_{c 2}\left(R_{o} G-1\right)}{R_{o} C_{2}^{q}}+\frac{v_{c 1}}{R_{o} C_{2}^{q}}-\frac{2 I_{s} e^{-\rho v_{c}} \sinh \left(\rho v_{c 2}\right)}{C_{2}^{q}}, \\
\frac{\mathrm{d}^{q} i_{1}}{\mathrm{~d} t^{q}}=\frac{v_{c 1}}{L_{1}^{q}}, \\
\frac{\mathrm{d}^{q} x}{\mathrm{~d} t^{q}}=F\left(x, v_{c 2}\right)=\left[\begin{array}{c}
\frac{2 I_{s}\left(e^{-\rho v_{c}} \cosh \left(\rho v_{c 2}\right)-1\right)-i_{L}}{C^{q}} \\
\frac{v_{c}-R i_{L}}{L^{q}}
\end{array}\right] .
\end{array}\right.
$$

The novel fractional-order RLC generalized memristive chaotic circuit model is established in MATLAB/Simulink, and numerical simulation is carried out on it. The system parameter values are set as $C_{1}^{q}=0.1 \mu \mathrm{F}, C_{2}^{q}=0.01 \mu \mathrm{F}$, $L_{1}^{q}=18 \mathrm{mH}, R_{o}=1.8 \mathrm{k} \Omega$, and $G=0.6667 \mathrm{mS}$. The parameters of the fractional-order generalized memristor remain unchanged. When the fractional order is 0.95 and the initial values of the state variables are set as $v_{c 1}=0 \mathrm{~V}, v_{c 2}=0.01 \mathrm{~V}$, $i_{1}=0 \mathrm{~A}, u_{c}=0 \mathrm{~V}$, and $i_{L}=0 \mathrm{~A}$, the phase diagrams of the fractional-order memristive chaotic circuit are shown in Figure 8.

\section{Dynamic Behavior Analysis of Fractional- Order Generalized Memristive Chaotic System}

3.1. Stability Analysis of the Equilibrium Point. The dynamic behavior of the proposed fractional-order memristive chaotic circuit is analyzed by calculating the equilibrium points and eigenvalues of the Jacobian matrix. The equilibrium points of the chaotic circuit can be obtained by solving the following equation:

$$
\left\{\begin{array}{l}
0=\frac{v_{c 2}-v_{c 1}}{R_{o} C_{1}^{q}}-\frac{i_{1}}{C_{1}^{q}}, \\
0=\frac{v_{c 2}\left(R_{o} G-1\right)}{R_{o} C_{2}^{q}}+\frac{v_{c 1}}{R_{o} C_{2}^{q}}-\frac{2 I_{s} e^{-\rho v_{c}} \sinh \left(\rho v_{c 2}\right)}{C_{2}^{q}}, \\
0=\frac{v_{c 1}}{L_{1}^{q}}, \\
0=\frac{2 I_{s}\left(e^{-\rho v_{c}} \cosh \left(\rho v_{c 2}\right)-1\right)-i_{L}}{C^{q}}, \\
0=\frac{v_{c}-R i_{L}}{L^{q}} .
\end{array}\right.
$$

Obviously, $Q_{1}(0,0,0,0,0)$ is an equilibrium point in the fractional-order RLC memristive chaotic circuit. This paper uses MATLAB-based graphical analysis methods to obtain other equilibrium points [34]. Simplify formula (10) to get

$$
\left\{\begin{array}{l}
v_{c 1}=0 \\
v_{c 2}=R i_{1} \\
v_{c 2}=\frac{2 R_{o} I_{s} e^{-p v_{c}} \sinh \left(p v_{c 2}\right)}{R_{o} G-1} \\
v_{c}=2 R I_{s}\left(e^{-p v_{c}} \cosh \left(p v_{c 2}\right)-1\right) .
\end{array}\right.
$$




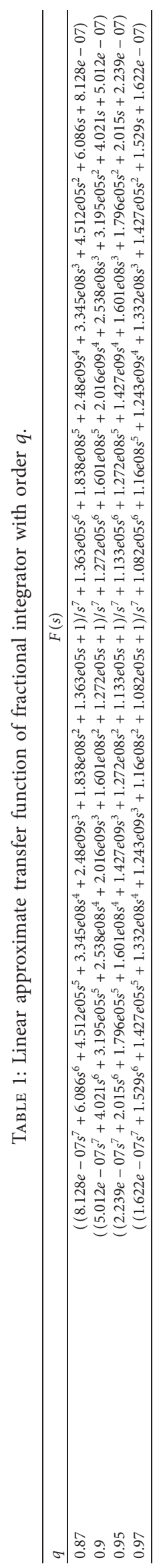




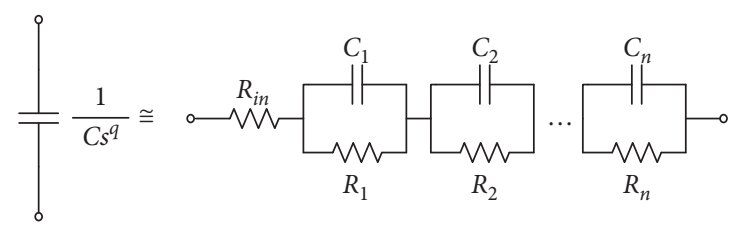

Figure 3: The equivalent unit circuit of the fractional capacitor $C^{q}$.

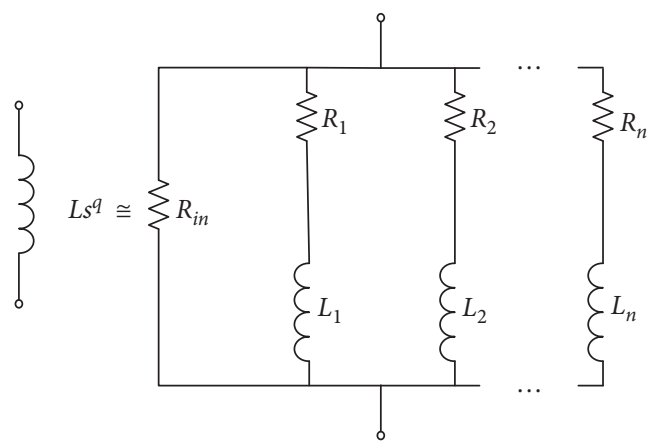

FIgURE 4: The equivalent unit circuit of the fractional inductor $L^{q}$.

TABLE 2: The calculated value of the resistance in the equivalent unit circuit of the fractional-order capacitor $C^{q}$.

\begin{tabular}{lcccccccc}
\hline$q$ & $R_{\text {in }}(\Omega)$ & $R_{1}(\Omega)$ & $R_{2}(\Omega)$ & $R_{3}(\Omega)$ & $R_{4}(\Omega)$ & $R_{5}(\Omega)$ & $R_{6}(\Omega)$ & $R_{7}(\Omega)$ \\
\hline 0.87 & 203 & 4,961 & $2.75 e 5$ & $1.51 e 7$ & $8.32 e 8$ & $4.57 e 10$ & $1.10 e 13$ & $6.63 e 6$ \\
0.9 & 125 & 2,882 & $1.84 e 5$ & $1.16 e 7$ & $7.31 e 8$ & $4.61 e 10$ & $1.56 e 13$ \\
0.95 & 56 & 905 & $7.26 e 4$ & $5.77 e 6$ & $4.58 e 8$ & $3.64 e 10$ & $2.78 e 13$ \\
0.97 & 40.5 & 451 & $3.97 e 4$ & $3.46 e 6$ & $3.01 e 8$ & $2.62 e 10$ & $3.5 e 13$ & $9.03 e 7$ \\
\hline
\end{tabular}

TABLE 3: The calculated value of the capacitance in the equivalent unit circuit of the fractional-order capacitor $C^{q}$.

\begin{tabular}{lccccccc}
\hline$q$ & $C_{1}(\mu \mathrm{F})$ & $C_{2}(\mu \mathrm{F})$ & $C_{3}(\mu \mathrm{F})$ & $C_{4}(\mu \mathrm{F})$ & $C_{5}(\mu \mathrm{F})$ & $C_{6}(\mu \mathrm{F})$ & $C_{7}(\mu \mathrm{F})$ \\
\hline 0.87 & $1.49 e-3$ & $2.69 e-3$ & $4.9 e-3$ & $8.91 e-3$ & $1.62 e-2$ & $1.33 e-2$ \\
0.9 & $2.76 e-3$ & $4.33 e-3$ & $6.85 e-3$ & $1.09 e-2$ & $1.72 e-2$ & $1.01 e-2$ \\
0.95 & $9.85 e-3$ & $1.23 e-2$ & $1.55 e-2$ & $1.95 e-2$ & $2.45 e-2$ & $6.34 e-3$ \\
0.97 & $2.07 e-2$ & $2.35 e-2$ & $2.7 e-2$ & $3.1 e-2$ & $3.56 e-2$ & $5.27 e-3$ & $1.96 e 3$ \\
\hline
\end{tabular}

TABLE 4: The calculated value of the resistance in equivalent cell circuit of fractional-order inductor $L^{q}$.

\begin{tabular}{|c|c|c|c|c|c|c|c|c|}
\hline$q$ & $R_{\text {in }}(\Omega)$ & $R_{1}(\Omega)$ & $R_{2}(\Omega)$ & $R_{3}(\Omega)$ & $R_{4}(\Omega)$ & $R_{5}(\Omega)$ & $R_{6}(\Omega)$ & $R_{7}(\Omega)$ \\
\hline 0.87 & $2.83 e 5$ & $1.16 e 4$ & $2.09 e 2$ & 3.8 & $6.91 e-2$ & $1.26 e-3$ & $5.21 e-6$ & 8.67 \\
\hline 0.9 & $4.59 e 5$ & $2.0 e 4$ & $3.13 e 2$ & 4.96 & $7.86 e-2$ & $1.25 e-3$ & $3.68 e-6$ & 2.32 \\
\hline 0.95 & $1.03 e 6$ & 0.637 & $2.07 e-6$ & $1.58 e-3$ & $1.26 e-1$ & 9.97 & 792 & $6.36 e 4$ \\
\hline 0.97 & $1.42 e 6$ & $1.27 e 5$ & $1.45 e 3$ & $1.66 e 1$ & $1.91 e-1$ & $2.19 e-3$ & $1.64 e-6$ & $4.19 e-1$ \\
\hline
\end{tabular}

TAвLe 5: The calculated value of inductance in equivalent cell circuit of fractional-order inductor $L^{q}$.

\begin{tabular}{lccccccc}
\hline$q$ & $L_{1}(\mathrm{H})$ & $L_{2}(\mathrm{H})$ & $L_{3}(\mathrm{H})$ & $L_{4}(\mathrm{H})$ & $L_{5}(\mathrm{H})$ & $L_{6}(\mathrm{H})$ & $L_{7}(\mathrm{H})$ \\
\hline 0.87 & $8.59 e-2$ & $1.548 e-1$ & $2.82 e-1$ & $5.13 e-1$ & $9.33 e-1$ & $7.65 e-1$ \\
0.9 & $1.58 e-1$ & $2.49 e-1$ & $3.94 e-1$ & $6.25 e-1$ & $9.9 e-1$ & $5.79 e-1$ & $3.27 e 6$ \\
0.95 & $1.12 e 5$ & 0.365 & 1.41 & 1.12 & 0.889 & 0.706 \\
0.97 & 1.19 & 1.35 & 1.55 & 1.7 & 2.05 & $3.03 e-1$ & $7.74 e 4$ \\
\hline
\end{tabular}



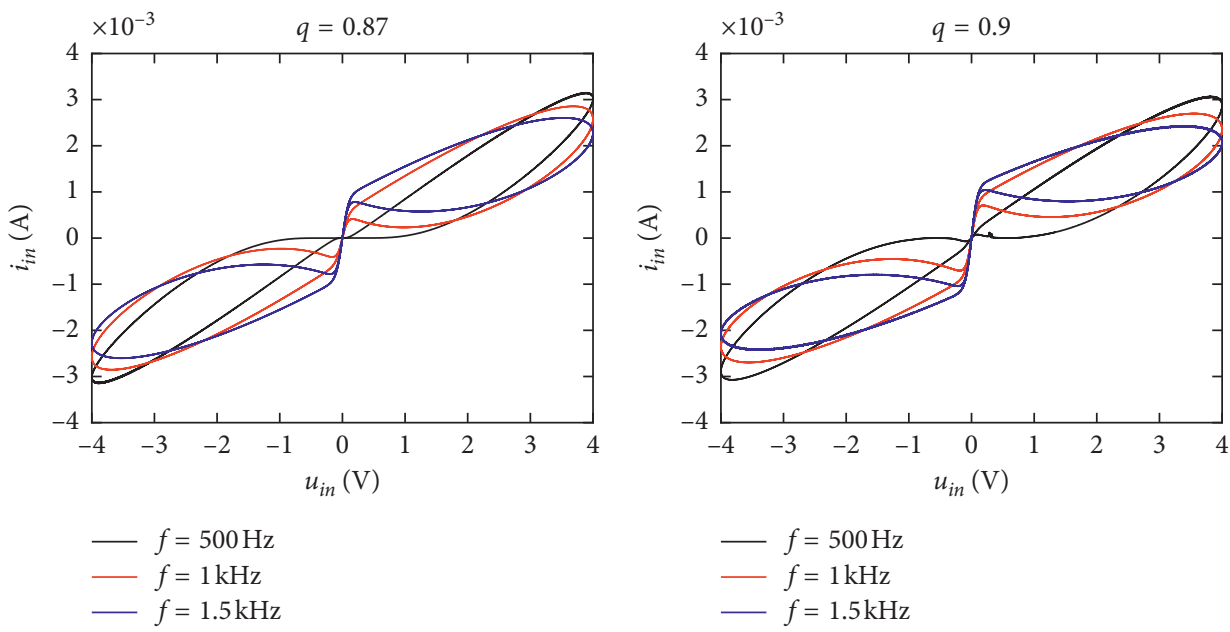

(a)

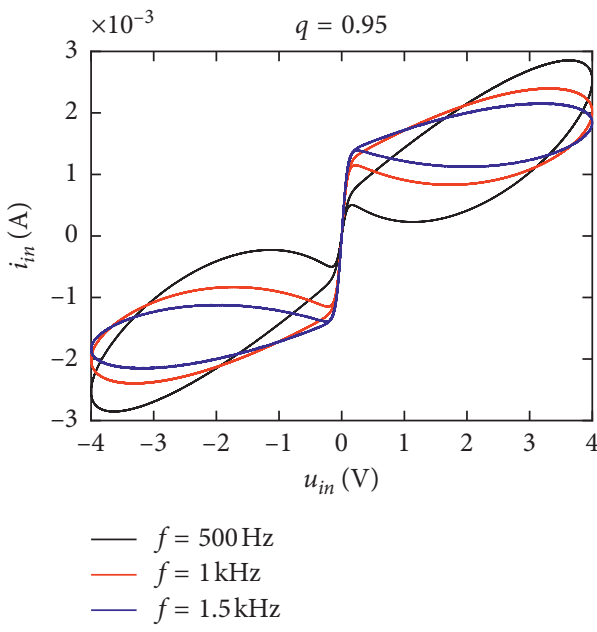

(c)

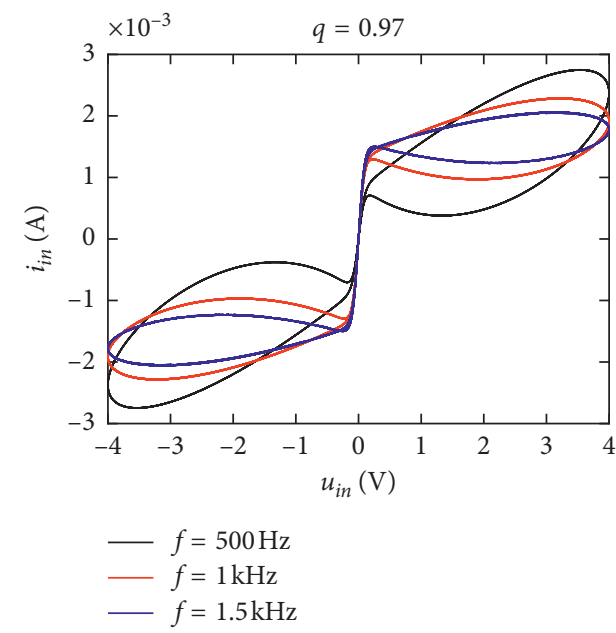

(d)

FiguRe 5: The tight hysteresis loop of the fractional generalized memristor under different orders. (a) $q=0.87$, (b) $q=0.9$, (c) $q=0.95$, and (d) $q=0.97$.

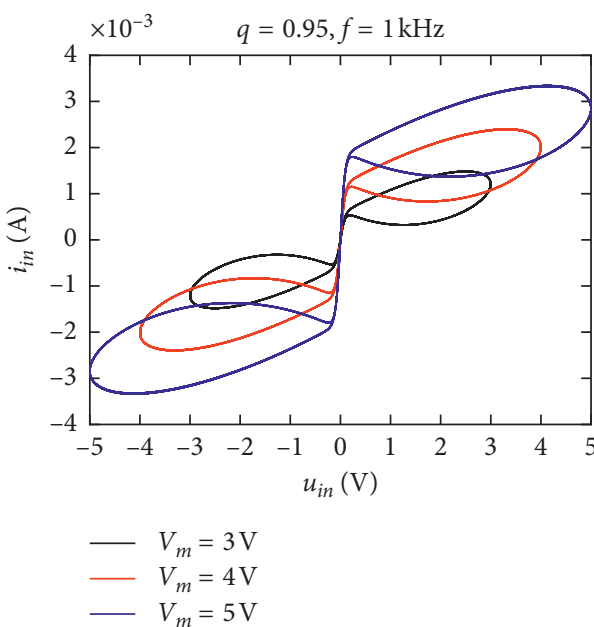

(a)

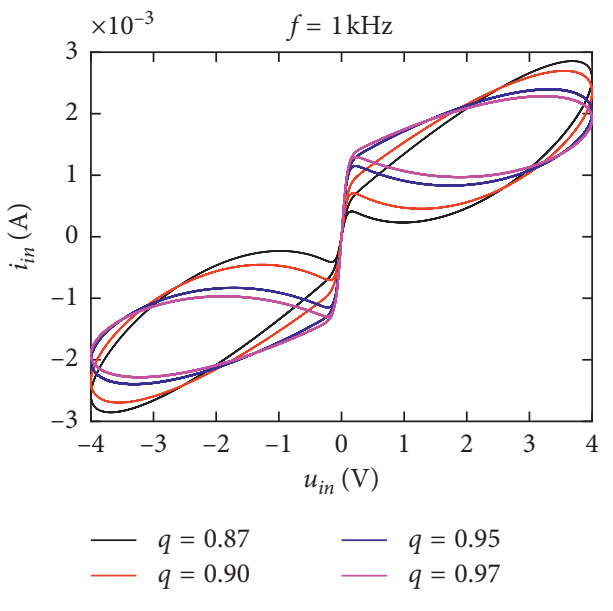

(b)

Figure 6: The tight hysteresis loop of the new fractional-order RLC generalized memristor. (a) Pinched hysteresis loop when $f=1 \mathrm{kHz}$, $q=0.95$, and $V_{m}$ varies; (b) pinched hysteresis loop when $f=1 \mathrm{kHz}, V_{m}=4 \mathrm{~V}$, and $q$ varies. 
Using MATLAB to plot the functions of $v_{c 2}$ and $v_{c}$, the coordinates of the intersection of the two functions are obtained, as shown in Figure 9. Therefore, the other two equilibrium points are $Q_{2,3}=(0, \pm 1.07478, \pm 0.000597$, 0.09556, 0.000119).

The Jacobian matrix of formula (9) is as follows:

$$
J=\left[\begin{array}{ccccc}
-a p & a p & -a & 0 & 0 \\
d p & f d-d p+b c d e^{-c v_{c}} \cosh \left(c v_{c 2}\right) & 0 & -b c d e^{-c v_{c}} \sinh \left(c v_{c 2}\right) & 0 \\
g & 0 & 0 & 0 & 0 \\
0 & b c m e^{-c v_{c}} \cosh \left(c v_{c 2}\right) & 0 & -b c m e^{-c v_{c}} \cosh \left(c v_{c 2}\right) & -m \\
0 & 0 & 0 & y & -z y
\end{array}\right]
$$

where $a=1 / C_{1}^{q}, b=2 I_{s}, c=\rho, d=1 / C_{2}^{q}, f=G, g=1 / L_{1}^{q}$, $m=1 / C^{q}, p=1 / R_{o}, y=1 / L$, and $z=R$. The eigenvalues of the chaotic system can be obtained by bringing the equilibrium point into the Jacobian matrix:

$$
\begin{aligned}
& \lambda_{1}^{\left(Q_{1}\right)}=16676.86 \\
& \lambda_{2}^{\left(Q_{1}\right)}=-5556.065+18427.691 i \\
& \lambda_{3}^{\left(Q_{1}\right)}=-5556.065-18427.691 i \\
& \lambda_{4}^{\left(Q_{1}\right)}=-1746.434+32923.507 i \\
& \lambda_{5}^{\left(Q_{1}\right)}=-1746.434-32923.507 i \\
& \lambda_{1}^{\left(Q_{2}\right)}=-184240.9 \\
& \lambda_{2}^{\left(Q_{2}\right)}=37723.12 \\
& \lambda_{3}^{\left(Q_{2}\right)}=-32043.28 \\
& \lambda_{4}^{\left(Q_{2}\right)}=-3704.807+19661.39 i \\
& \lambda_{5}^{\left(Q_{2}\right)}=-3704.807-19661.39 i \\
& \lambda_{1}^{\left(Q_{3}\right)}=-385909.9 \\
& \lambda_{2}^{\left(Q_{3}\right)}=211961.5 \\
& \lambda_{3}^{\left(Q_{3}\right)}=-3352.592+23299.84 i \\
& \lambda_{4}^{\left(Q_{3}\right)}=-3352.592-23299.84 i \\
& \lambda_{5}^{\left(Q_{3}\right)}=-5317.097
\end{aligned}
$$

From the above eigenvalues, it can be seen that all three equilibrium points have a positive real root, so the three equilibrium points are the unstable saddle points of the system.

\subsection{Bifurcation Behaviors of Fractional-Order Memristive} Chaotic System. In order to study the dynamic influence of the capacitance $C^{q}$ value on the fractional memristive chaotic system, the initial value of the chaotic system is set to $(0 \mathrm{~V}, 0.01 \mathrm{~V}, 0 \mathrm{~A}, 0 \mathrm{~V}, 0 \mathrm{~A})$, and the bifurcation diagram of the fractional-order memristive chaotic system can be obtained, as shown in Figure 10. It can be seen from Figure 10 that the dynamic behaviors of the fractional-order memristive chaotic system have three states: period, bifurcation, and chaos, and a narrow period window appears in the chaotic state. When $C^{q}<0.8 \mathrm{nF}$, the fractional-order system is in a single cycle state. As the value of $C^{q}$ increases, the system enters complex nonlinear dynamic behaviors, such as bifurcation and chaos. When $0.8 \mathrm{nF}<C^{q}<0.98 \mathrm{nF}$, the fractional-order system is in cycle 2 state. When $1.55 \mathrm{nF}<C^{q}<1.65 \mathrm{nF}$, the system is in a narrow periodic window in the chaotic region. Figure 11 shows 


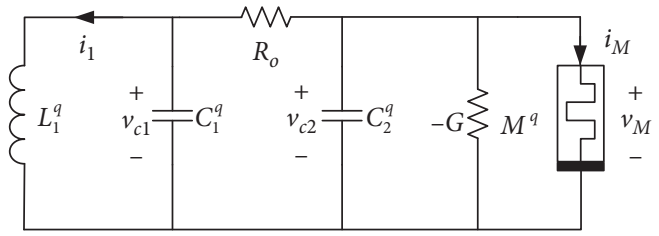

Figure 7: The fractional Chua's memristive chaotic circuit.

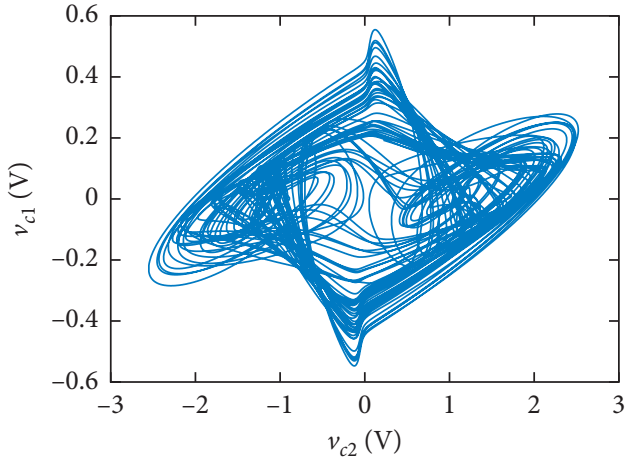

(a)

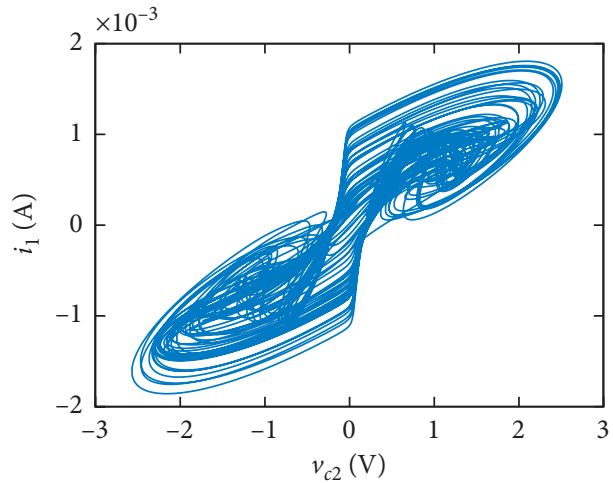

(c)

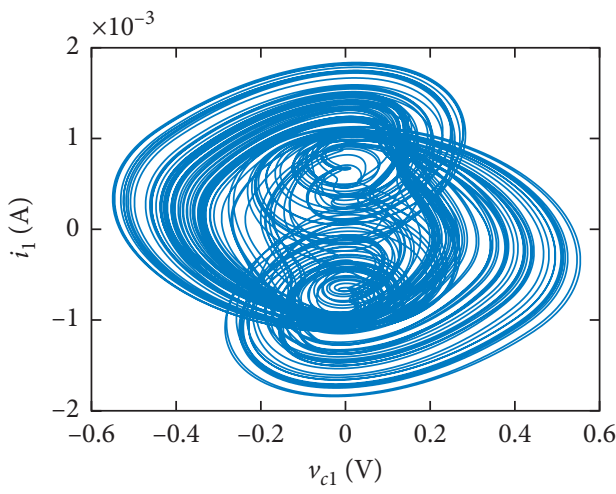

(b)

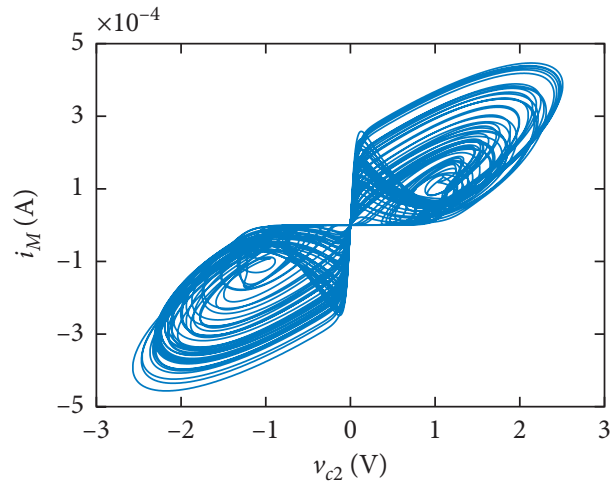

(d)

FIGURE 8: Phase diagrams of fractional-order memristive chaotic circuit. (a) Phase trajectory in $v_{c 2}-v_{c 1}$ plane; (b) phase trajectory in $v_{c 1}-i_{1}$ plane; (c) phase trajectory in $v_{c 2}-i_{1}$ plane; (d) phase trajectory in $v_{c 2}-i_{M}$ plane.

the phase diagrams of the fractional-order memristive chaotic circuit with different values of the capacitor $C^{q}$. The results of the phase diagrams are consistent with the dynamic behaviors described in the bifurcation diagram. As shown in Figure 11(a), the fractional-order memristive chaotic system is in a periodic state when $C^{q}=0.76 \mathrm{nF}$. As can be seen in Figure 11(b), the system is in a bifurcated state, and the cycle 2 state appears when $C^{q}=0.9 \mathrm{nF}$. When $C^{q}=1.6 \mathrm{nF}$, the system is in a narrow periodic state in the chaotic region as shown in Figure $11(\mathrm{c})$. When $C^{q}=4 \mathrm{nF}$, the system is in a chaotic state, as shown in Figure 11(d).

\section{Circuit Simulation Experiment of the Fractional-Order Memristive Chaotic System}

The same equivalent circuits are used to realize the capacitors $C_{1}^{q}$ and $C_{2}^{q}$ and inductor $L^{q}$ in the fractional-order memristive chaotic circuit. In the whole system, the value of order $q$ is 0.95 . Tables 6 and 7 show the equivalent resistance, capacitance, and inductance parameters of the fractional-order capacitors $C_{1}^{q}$ and $C_{2}^{q}$ and the fractionalorder inductor $L^{q}$. The simulation diagram of the fractional-order Chua's memristive circuit is shown in 


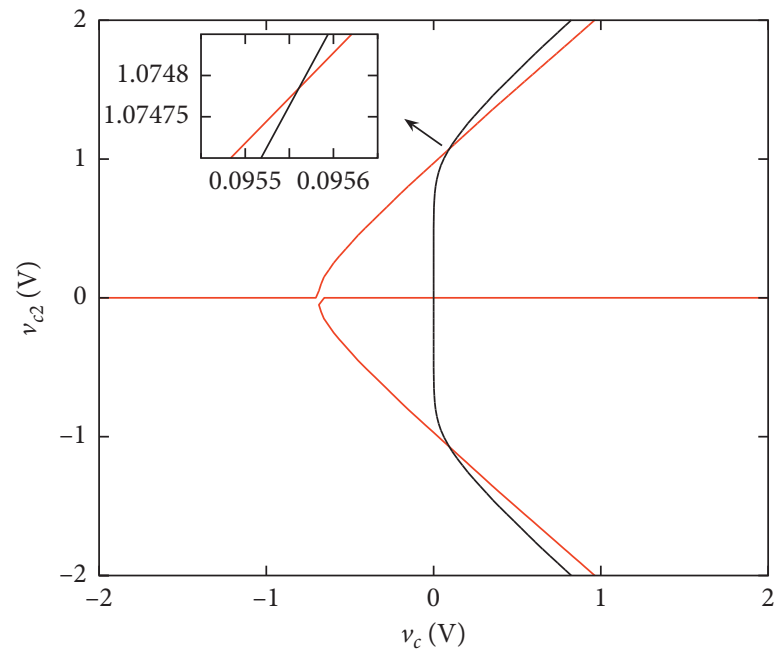

Figure 9: Plotted functions and their intersection points.

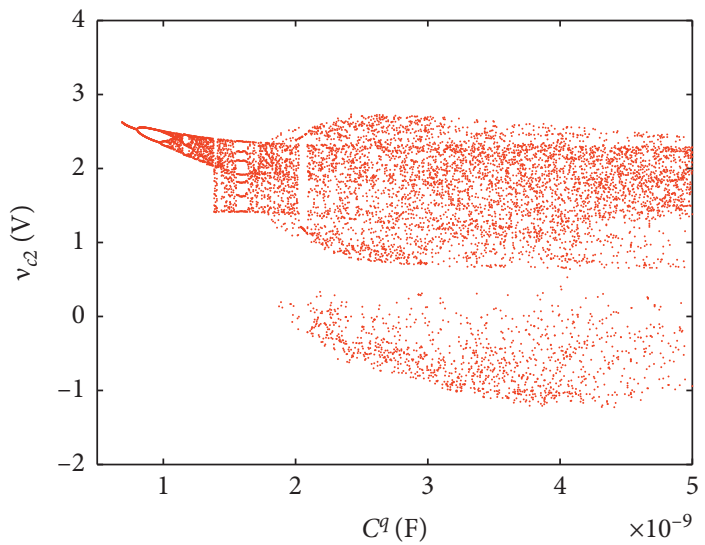

FIgURE 10: The bifurcation diagram of the fractional-order memristive chaotic circuit with capacitor $C^{q}$ as a parameter.
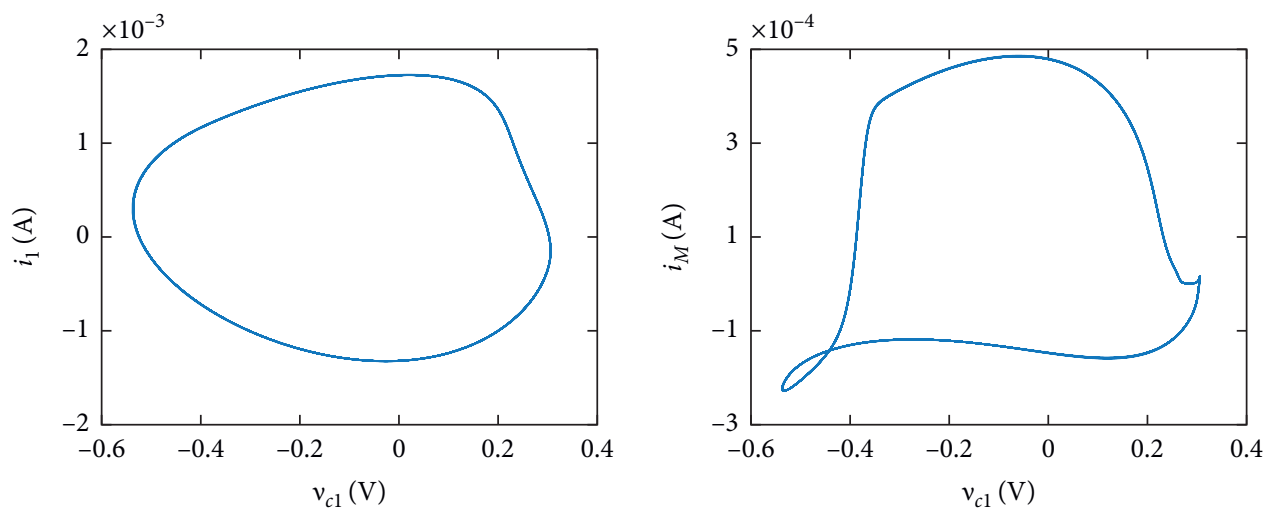

(a)

FIgURE 11: Continued. 

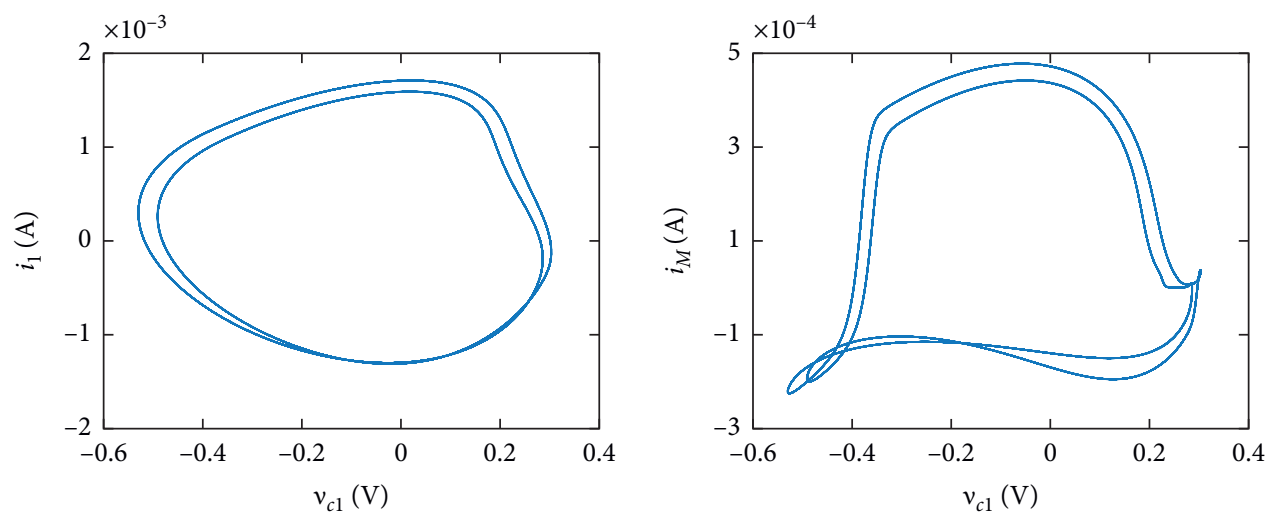

(b)
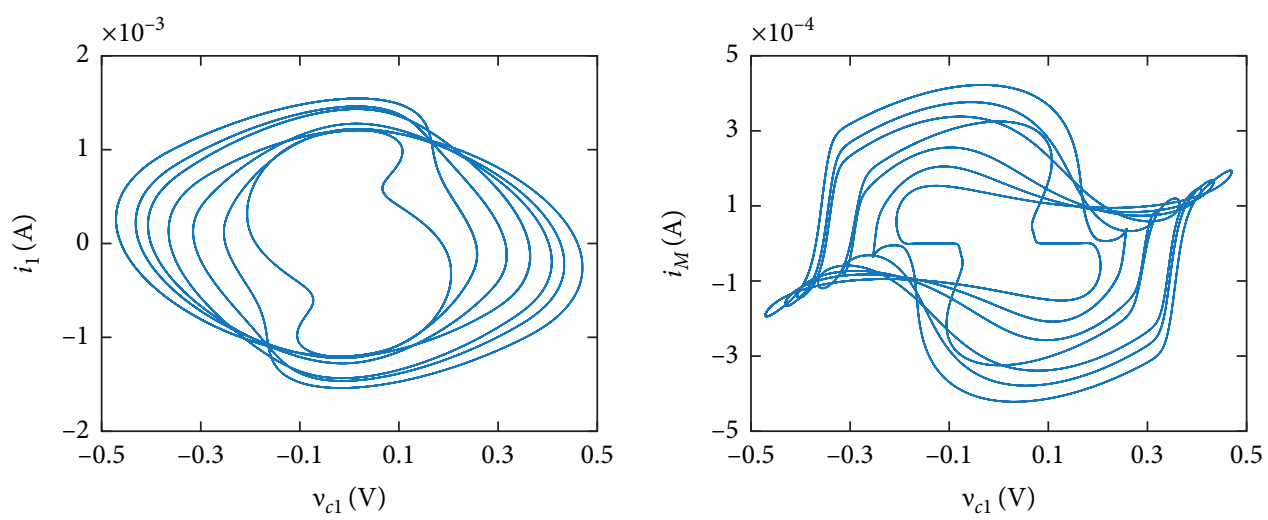

(c)
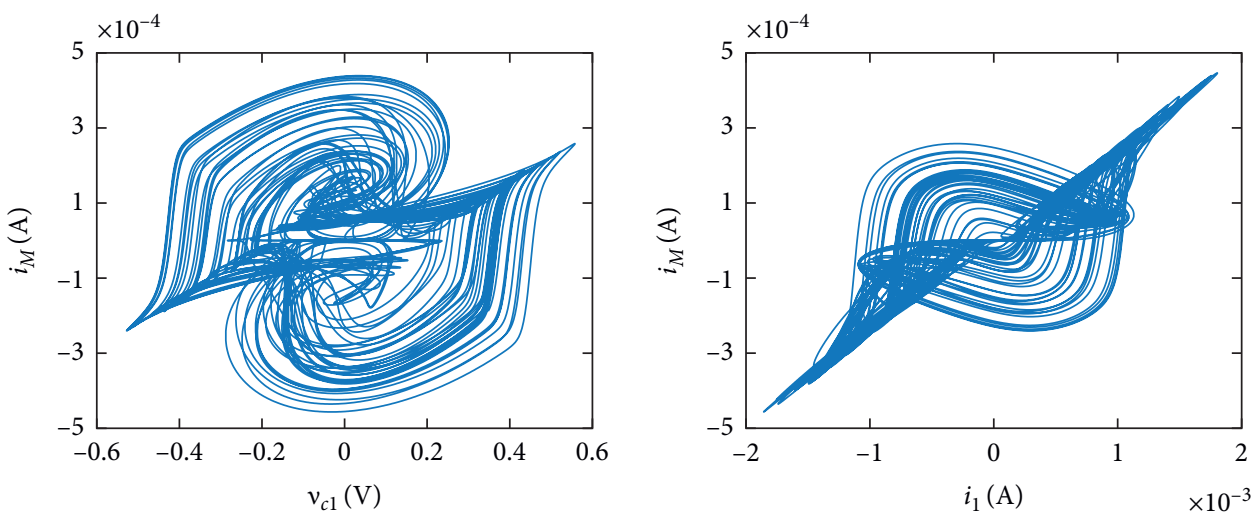

(d)

FIGURE 11: Phase diagrams of the fractional-order memristive chaotic circuit with different values of capacitance $C^{q}$. (a) When $C^{q}=0.76 \mathrm{nF}$, phase trajectories in $v_{c 1}-i_{1}$ and $v_{c 1}-i_{M}$ plane; (b) when $C^{q}=0.9 \mathrm{nF}$, phase trajectories in $v_{c 1}-i_{1}$ and $v_{c 1}-i_{M}$ plane; (c) when $C^{q}=1.6 \mathrm{nF}$, phase trajectories in $v_{c 1}-i_{1}$ and $v_{c 1}-i_{M}$ plane; (d) when $C^{q}=4 \mathrm{nF}$, phase trajectories in $v_{c 1}-i_{M}$ and $i_{1}-i_{M}$ plane.

Figure 12. The diode in the chaotic circuit is set with the parameters of $1 \mathrm{~N} 4148$, and the values of $R_{o}, R$, and $L^{q}$ remain unchanged.
Figure 13 shows the phase diagrams of the fractionalorder memristive chaotic circuit in the chaotic state when $C^{q}=4 \mathrm{nF}$. The results verify the correctness of the numerical 
TABLE 6: The equivalent resistance parameters of fractional-order capacitors $C_{1}^{q}$ and $C_{2}^{q}$ and inductor $L_{1}^{q}$ when $q=0.95$.

\begin{tabular}{cccccccc}
\hline & $R_{\text {in }}(\Omega)$ & $R_{1}(\Omega)$ & $R_{2}(\Omega)$ & $R_{3}(\Omega)$ & $R_{4}(\Omega)$ & $R_{5}(\Omega)$ & $R_{6}(\Omega)$ \\
\hline$L_{1}^{q}$ & $8.04 e 4$ & 4,970 & 62 & 0.78 & $9.83 e-3$ & $1.24 e-4$ & $1.62 e-7$ \\
$C_{1}^{q}$ & 2.24 & 36.2 & $2.9 e 3$ & $2.31 e 5$ & $1.83 e 7$ & $1.46 e 9$ & $1.11 e 12$ \\
$C_{2}^{q}$ & $2.24 e 1$ & $3.62 e 2$ & $2.90 e 4$ & $2.31 e 6$ & $1.83 e 8$ & $1.46 e 10$ & $1.11 e 13$ \\
\hline
\end{tabular}

TABLE 7: The equivalent capacitance and inductance parameters of fractional-order capacitors $C_{1}^{q}$ and $C_{2}^{q}$ and inductor $L_{1}^{q}$ when $q=0.95$.

\begin{tabular}{cccccccc}
\hline & $L_{1}(\mathrm{H})$ & $L_{2}(\mathrm{H})$ & $L_{3}(\mathrm{H})$ & $L_{4}(\mathrm{H})$ & $L_{5}(\mathrm{H})$ & $L_{6}(\mathrm{H})$ & $L_{7}(\mathrm{H})$ \\
\hline$L_{1}^{q}$ & $4.43 e-2$ & $5.53 e-2$ & $6.96 e--2$ & 0.0876 & 0.11 & 0.0285 \\
& $C_{1}(\mu \mathrm{F})$ & $C_{2}(\mu \mathrm{F})$ & $C_{3}(\mu \mathrm{F})$ & $C_{4}(\mu \mathrm{F})$ & $C_{5}(\mu \mathrm{F})$ & $C_{6}(\mu \mathrm{F})$ & $C_{7}(\mu \mathrm{F})$ \\
$C_{1}^{q}$ & $2.46 e-1$ & $3.07 e-1$ & $3.86 e-1$ & $4.86 e-1$ & $6.12 e-1$ & $1.59 e-1$ & $4.89 e 4$ \\
$C_{2}^{q}$ & $2.46 e-2$ & $3.07 e-2$ & $3.86 e-2$ & $4.86 e-2$ & $6.12 e-2$ & $1.59 e-2$ & $4.89 e 3$ \\
\hline
\end{tabular}

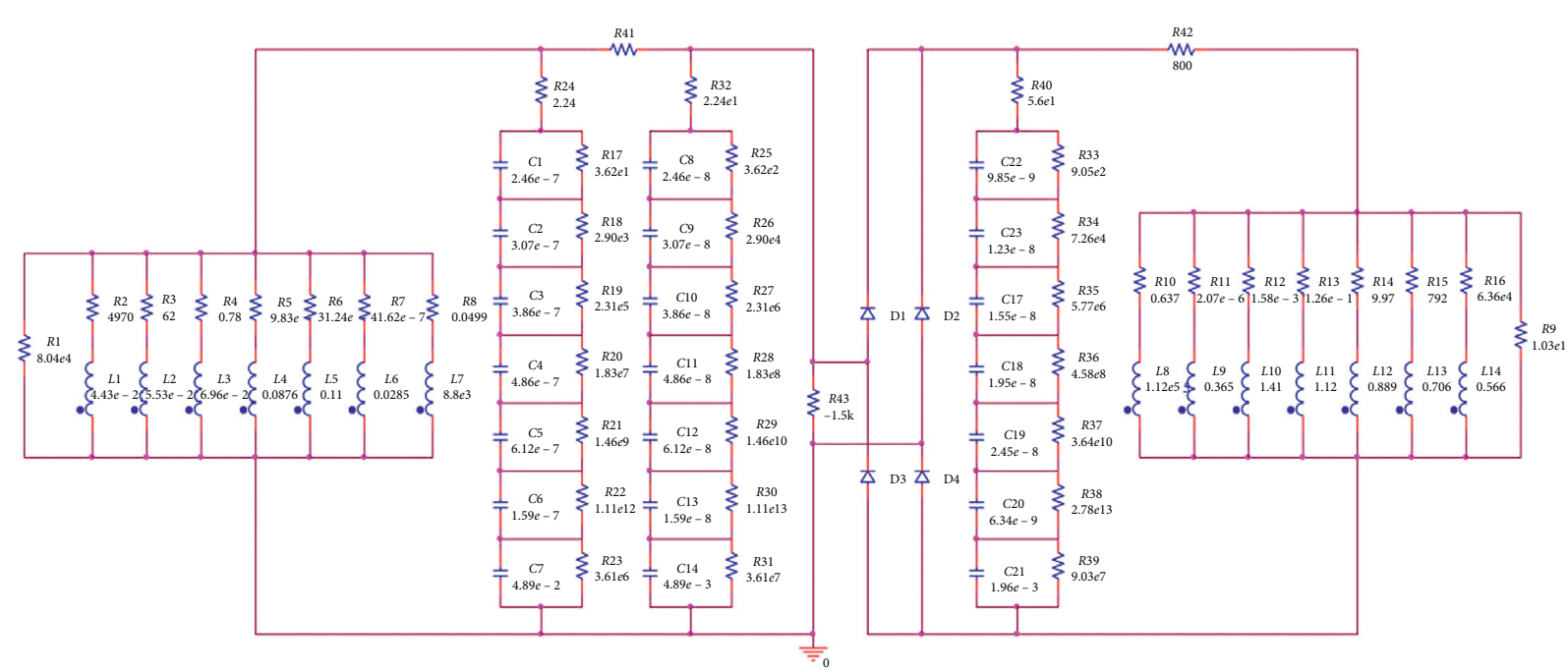

FIGURE 12: The equivalent circuit of the fractional-order Chua's memristive chaotic system.

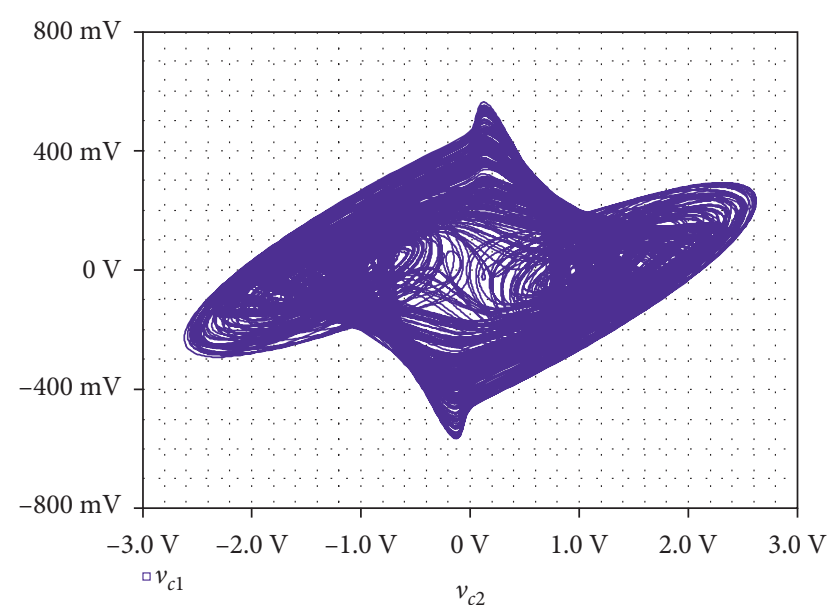

(a)

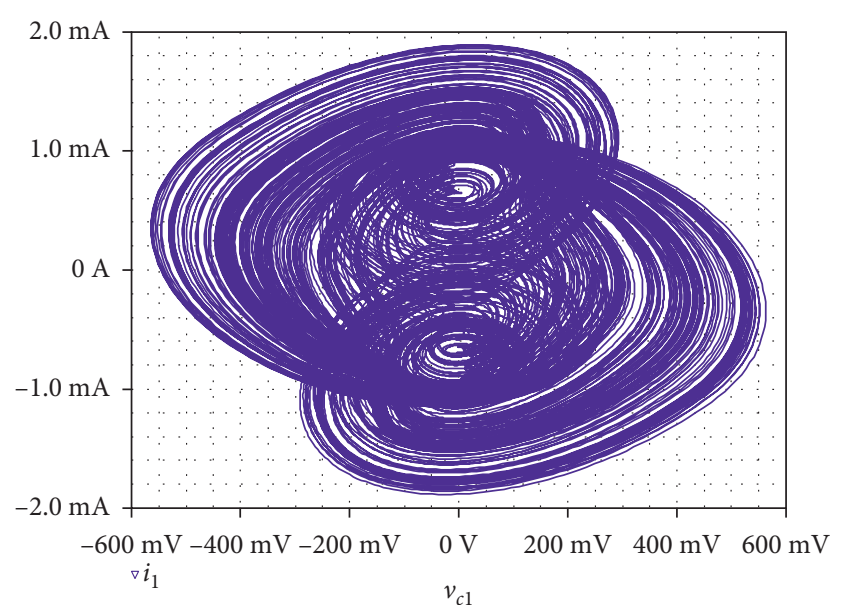

(b)

Figure 13: Continued. 


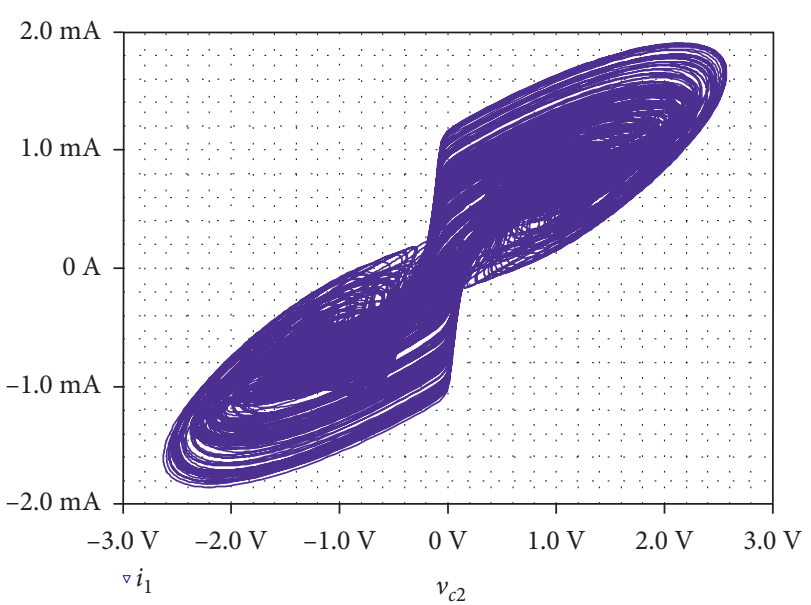

(c)

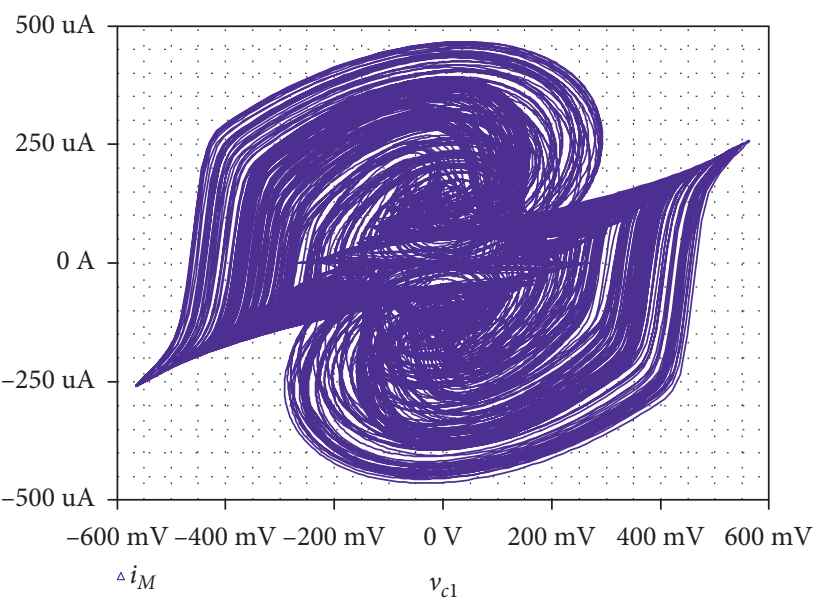

(e)

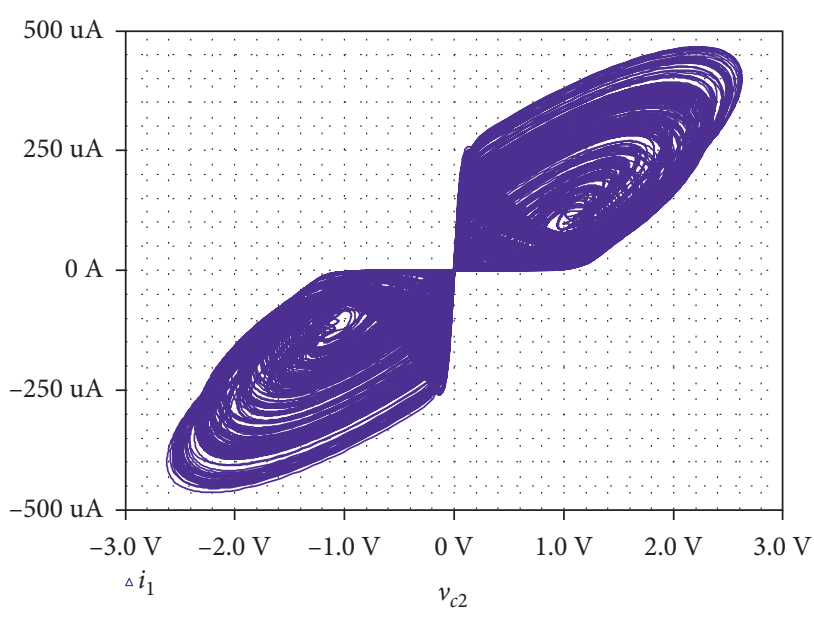

(d)

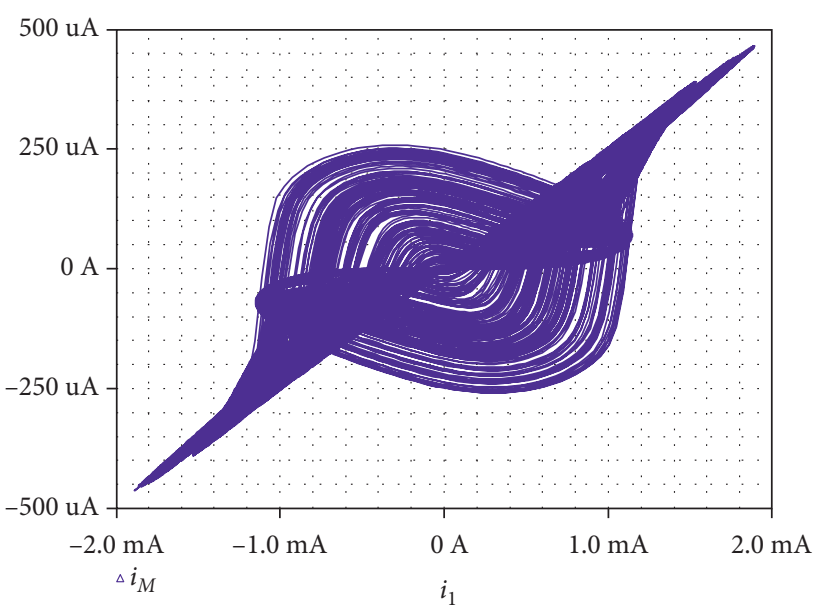

(f)

Figure 13: Chaotic phase diagrams in circuit simulation based on PSpice software. (a) Phase trajectory in $v_{c 2}-v_{c 1}$ plane; (b) phase trajectory in $v_{c 1}-i_{1}$ plane; (c) phase trajectory in $v_{c 2}-i_{1}$ plane; (d) phase trajectory in $v_{c 2}-i_{M}$ plane; (e) phase trajectory in $v_{c 1}-i_{M}$ plane; (f) phase trajectory in $i_{1}-i_{M}$ plane.

simulations and the practicality of the fractional-order capacitor and inductor equivalent circuit.

\section{Conclusions}

This paper proposes a fractional-order generalized memristor composed of a fractional-order capacitor, a fractionalorder inductor, a resistor, and diodes and analyzes and verifies the memristive characteristics. Then Chua's chaotic circuit is extended to the fractional order and combined with the fractional-order generalized memristor to form a fractional-order Chua's memristive chaotic circuit. The numerical simulation and circuit simulation of the fractionalorder Chua's memristive chaotic circuit show that the three equilibrium points of the proposed fractional-order chaotic system are the unstable saddle points of the system, and the value of the capacitance $C^{q}$ has an effect on the dynamic behaviors of the fractional-order chaotic system significantly. As the value of capacitance $C^{q}$ increases, the fractional-order Chua's memristive chaotic circuit enters a bifurcation from a periodic state and finally enters chaos state. A narrow period window was found in the chaotic state. The phase diagrams in the corresponding state are given in the paper, which are consistent with the dynamic characteristics described in the bifurcation diagram. Finally, the equivalent circuit of the fractional-order generalized memristive chaotic circuit is built in PSpice, and the results obtained by circuit simulation are consistent with the theoretical analysis and numerical simulations.

\section{Data Availability}

The data used to support the findings of this study are available from the corresponding author upon request. 


\section{Conflicts of Interest}

The authors declare that there are no conflicts of interest regarding the publication of this study.

\section{Acknowledgments}

This work was supported by the National Natural Science Foundation of China (Grant no. 51507134) and Natural Science Foundation of Shaanxi Province (Grant nos. 2018JM5068 and 2021JM-449).

\section{References}

[1] F. W. Hu, B. C. Bao, H. G. Wu, and C. L. Wang, "Equivalent circuit analysis model of charge-controlled memristor and its circuit characteristics," Acta Physica Sinica, vol. 62, no. 21, pp. 404-411, 2013.

[2] D. B. Strukov, G. S. Snider, D. R. Stewart, and R. S. Williams, "The missing memristor found," Nature, vol. 453, no. 7191, pp. 80-83, 2008.

[3] B. Bao, L. Xu, N. Wang, H. Bao, Q. Xu, and M. Chen, “Thirdorder RLCM-four-elements-based chaotic circuit and its coexisting bubbles," AEU-International Journal of Electronics and Communications, vol. 94, pp. 26-35, 2018.

[4] J. Ruan, K. Sun, J. Mou, S. He, and L. Zhang, "Fractional-order simplest memristor-based chaotic circuit with new derivative," The European Physical Journal Plus, vol. 133, no. 1, p. 3, 2018.

[5] T. S. Wang, R. D. Zhang, Z. Guan, K. Ba, and Y. X. Zu, "Properties of memristor in RLC circuit and diode circuit," Acta Physica Sinica, vol. 63, no. 17, pp. 337-344, 2014.

[6] P. Srivastava, R. K. Gupta, R. K. Sharma, and R. K. Ranjan, "MOS-only memristor emulator," Circuits, Systems, and Signal Processing, vol. 39, no. 11, pp. 5848-5861, 2020.

[7] M. Itoh and L. O. Chua, "Memristor oscillators," International Journal of Bifurcation and Chaos, vol. 18, no. 11, pp. 31833206, 2008.

[8] B. C. Bao Bo-Cheng, Z. Liu Zhong, and B. P. Xu Jian-Ping, "Dynamical analysis of memristor chaotic oscillator," Acta Physica Sinica, vol. 59, no. 6, pp. 3785-3793, 2010.

[9] S. Vaidyanathan, V. T. Pham, C. K. Volos, T. P. Le, and V. Y. Vu, "A memristor-based hyperchaotic system with hidden attractors: dynamics, synchronization and circuital emulating," Journal of Engineering Science and Technology Review, vol. 8, no. 2, pp. 205-214, 2015.

[10] N. Wang, B. C. Bao, T. Jiang, M. Chen, and Q. Xu, "Parameter-independent dynamical behaviors in memristorbased wien-bridge oscillator," Mathematical Problems in Engineering, vol. 2017, Article ID 5897286, 13 pages, 2017.

[11] M. E. Sahin, A. S. Demirkol, H. Guler, and S. E. Hamamci, "Design of a hyperchaotic memristive circuit based on wien bridge oscillator," Computers and Electrical Engineering, vol. 88, Article ID 106826, 2020.

[12] H. Chang, Y. X. Li, G. R. Chen, and F. Yuan, "Extreme multistability and complex dynamics of a memristor-based chaotic system," International Journal of Bifurcation and Chaos, vol. 30, no. 8, Article ID 2030019, 2020.

[13] Y. X. Song, F. Yuan, and Y. X. Li, "Coexisting attractors and multistability in a simple memristive wien-bridge chaotic circuit," Entropy, vol. 21, no. 7, Article ID 678, 2019.

[14] X. Zhang, C. B. Li, Y. D. Chen, H. C. Herbert, and T. F. Lei, "A memristive chaotic oscillator with controllable amplitude and frequency," Chaos Solitons \& Fractals, vol. 139, Article ID 110000, 2020.

[15] G. Dou, H. Yang, Z. Gao, and P. Li, "Coexisting multi-dynamics of a physical SBT memristor-based chaotic circuit," International Journal of Bifurcation and Chaos, vol. 30, no. 11, Article ID 2030043, 2020.

[16] Q. Yu, B. C. Bao, F. W. Hu, Q. Xu, M. Chen, and J. Wang, "Wien-bridge chaotic oscillator based on fisrt-order generalized memristor," Acta Physica Sinica, vol. 63, no. 24, Article ID 240505, 2014.

[17] B. C. Bao, J. J. Yu, F. W. Hu, and Z. Liu, "Generalized memristor consisting of diode bridge with first order parallel RC filter," International Journal of Bifurcation and Chaos, vol. 24, no. 11, Article ID 1450143, 2014.

[18] G. Carlson and C. Halijak, "Approximation of fractional capacitors $(1 / \mathrm{s})(1 / \mathrm{n})$ by a regular Newton process," IEEE Transactions on Circuit Theory, vol. 11, no. 2, pp. 210-213, 2003.

[19] N. N. Yang, C. Xu, C. J. Wu, R. Jia, and C. X. Liu, "Modeling and analysis of a fractional-order generalized memristorbased chaotic system and circuit implementation," International Journal of Bifurcation and Chaos, vol. 27, no. 13, Article ID 1750199, 2017.

[20] D. W. Ding, J. Luo, X. Y. Shan, Y. B. Hu, Z. L. Yang, and L. H. Ding, "Coexistent multiple-stability of a fractional-order delayed memristive Chua's system based on describing function," Modern Physics Letters B, vol. 34, no. 14, Article ID 2050146, 2020.

[21] B. Yan, S. B. He, and S. J. Wang, "Multistability and formation of spiral waves in a fractional-order memristor-based hyperchaotic lü system with No equilibrium points," Mathematical Problems in Engineering, vol. 2020, Article ID 2468134, 12 pages, 2020.

[22] I. Petras, "Fractional-order memristor-based Chua's circuit," IEEE Transactions on Circuits and Systems II: Express Briefs, vol. 57, no. 12, pp. 975-979, 2010.

[23] J. Wu, G. Y. Wang, H. H. C. Iu, Y. R. Shen, and W. Zhou, “A nonvolatile fractional order memristor model and its complex dynamics," Entropy, vol. 21, no. 10, 2019.

[24] M. E. Fouda and A. G. Radwan, "Fractional-order memristor response under DC and periodic signals," Circuits, Systems, and Signal Processing, vol. 34, no. 3, pp. 961-970, 2015.

[25] Y. J. Yu, H. Bao, M. Shi, B. C. Bao, Y. Q. Chen, and M. Chen, "Complex dynamical behaviors of a fractional-order system based on a locally active memristor," Complexity, vol. 2019, Article ID 2051053, 13 pages, 2019.

[26] D. Cafagna and G. Grassi, "On the simplest fractional-order memristor-based chaotic system," Nonlinear Dynamics, vol. 70, no. 2, pp. 1185-1197, 2012.

[27] M. S. Semary, H. L. Abdel Malek, H. N. Hassan, and A. G. Radwan, "An optimal linear system approximation of nonlinear fractional-order memristor-capacitor charging circuit," Microelectronics Journal, vol. 51, pp. 58-66, 2016.

[28] Y. Y. Wang, L. Liu, X. S. Cai, C. X. Liu, Y. Wang, and G. C. Zheng, "Fractional-order hidden attractor based on the extended liu system," Mathematical Problems in Engineering, vol. 2020, Article ID 1418272, 22 pages, 2020.

[29] K. B. Oldham and J. Spanier, "The fractional calculus," Mathematical Gazette, vol. 56, no. 247, pp. 396-400, 1974.

[30] F. Corinto and A. Ascoli, "Memristive diode bridge with LCR filter," Electronics Letters, vol. 48, no. 14, pp. 824-825, 2012.

[31] A. Oustaloup, F. Levron, and B. Mathieu, "Frequency-band complex noninteger differentiator: characterization and synthesis," IEEE Transactions on Circuits \& Systems I 
Fundamental Theory \& Applications, vol. 47, no. 1, pp. 25-39, 2002.

[32] S. P. Adhikari, M. P. Sah, H. Kim, and L. O. Chua, "Three fingerprints of memristor," IEEE Transactions on Circuits and Systems I: Regular Papers, vol. 60, no. 11, pp. 3008-3021, 2013.

[33] N. N. Yang, C. Xu, C. J. Wu, R. Jia, and C. X. Liu, "Dynamic behaviors and the equivalent realization of a novel fractionalorder memristor-based chaotic circuit," Complexity, vol. 2018, Article ID 9467435, 13 pages, 2018.

[34] M. Chen, M. Y. Li, Q. Yu, B. C. Bao, Q. Xu, and J. Wang, "Dynamics of self-excited attractors and hidden attractors in generalized memristor-based Chua's circuit," Nonlinear Dynamics, vol. 81, no. 1-2, pp. 215-226, 2015. 